\title{
Abundancia Relativa del Colibrí Esmeralda (Amazilia luciae) en su Comunidad de Aves en el Valle de Agalta, Olancho, Honduras
}

\author{
José Manuel Mora', Mario Roberto Espinal ${ }^{2}$, Luis Daniel Germer ${ }^{3}$ y Lucía Isabel López ${ }^{4}$
}

Resumen. El colibrí esmeralda hondureño (Amazilia luciae) es la única ave endémica de Honduras. Esta especie ha sido catalogada como una de las aves más amenazadas de Centroamérica. El colibrí esmeralda existe solamente en seis áreas de bosque seco de Honduras. En el oriente se conoce de los valles de Agalta y Telica (Olancho) y el valle de Aguán (Yoro). En el occidente se ha observado en los valles de Tencoa, Quimistán y Jicatuyo (Santa Bárbara y Cortés). El hábitat de bosque seco del valle de Agalta ha sido eliminado y deteriorado y para el 2102 solo permanecían 20 fragmentos de diferentes tamaños. En estos fragmentos censamos las aves con énfasis en el colibrí esmeralda. Hicimos 50 transectos de punto y categorizamos a las aves según su abundancia. El colibrí esmeralda resultó ser relativamente común en el área. Lo encontramos en 10 de los fragmentos de bosque seco de Agalta. Esta fue la única especie especialista de las 90 que detectamos en el valle. Las demás especies son generalistas y la mayoría comunes o abundantes. Una de las especies comunes fue Arremonops chloronotus twomeyi, una subespecie que solo existe en los valles secos de Agalta y Aguán. A pesar de que observamos al colibrí, principalmente en los bordes de bosque seco, el mayor problema para su conservación en Agalta es la tenencia de la tierra. Diecinueve de los remanentes de bosque seco en Agalta son propiedad privada, el otro es municipal. Planteamos algunas alternativas para la conservación del colibrí esmeralda en Agalta. De estas, la que podría ser más factible es la creación de un programa de pagos por servicios ambientales (PSA). La regeneración del bosque en potreros en desuso actualmente no es una solución a largo plazo si siguen en manos privadas. Los propietarios privados protegerán el bosque si reciben estímulos económicos para hacerlo. Un PSA podría obtener parte de su financiamiento para operar por medio de un peaje ambiental en la carretera Gualaco-San Esteban.

Palabras clave: Bosque seco tropical, censos de aves, especies raras, fragmentos de bosque, valles áridos.

\section{Relative abundance of the Honduran Emerald (Amazilia luciae) and its Bird Community in the Agalta Valley, Olancho, Honduras}

\begin{abstract}
The Honduran Emerald (Amazilia luciae) is the only bird endemic to Honduras. This species has been categorized as one of the most threatened birds in Central America. The Honduran Emerald exists only in six dry forest areas of Honduras. It is known from Agalta and Telica valleys (Olancho) and Aguan valley (Yoro) in the east. It has been observed in Tencoa, Quimistan, and Jicatuyo valleys (Santa Barbara and Cortes) in the west. Dry forest habitat in Agalta Valley has been eliminated and deteriorated and only 20 fragments of different sizes remained in 2012. We conducted bird census in these fragments with emphasis on the emerald hummingbird. We counted birds in 50 transept points and categorize birds by abundance. The emerald hummingbird turned out to be relatively common in the area. We found the species in 10 of the Agalta dry forest fragments. This was the only specialist species out of the 90 that we find in the valley. Other species are generalists and common or abundant. One of the common species was Arremonops chloronotus twomeyi, a subspecies that only exists in the dry valleys of Agalta and Aguán. Although we observe the hummingbird mainly on the edges of dry forest, the biggest problem for conservation in Agalta is land tenure. Nineteen of the Agalta dry forest fragments are privately owned, the other one is municipal. We propose some alternatives to conserve the emerald hummingbird in Agalta. Of these, it might be that the creation of a program of payments for environmental services (PES) is the most

\footnotetext{
${ }^{1}$ Instituto Internacional en Conservación y Manejo de Vida Silvestre (ICOMVIS), Universidad Nacional (UNA), Heredia, Costa Rica. Email josemora07@gmail.com

2 Investigador Asociado, Centro Zamorano de Biodiversidad, Escuela Agrícola Panamericana, Zamorano, Honduras. Email mknorops@gmail.com

${ }^{3}$ Biólogo Especialista en Avifauna Hondureña. Barrio La leona, Tegucigalpa M.D.C. Email germer.daniel@gmail.com

${ }^{4}$ Bióloga y consultora ambiental, Cinco Esquinas, Carrizal, Alajuela, Costa Rica. Email luciaisa2@gmail.com
} 
feasible. Forest regeneration in pastures not in use is not a long term solution if they still are in private hands. Private owners will protect the forest if they receive economic incentives to do so. A PES could get some of its funding to operate through an "environmental toll" on the Gualaco-San Esteban road.

Key words: Arid valleys, bird census, forest fragments, rare species, tropical dry forest.

\section{Introducción}

El colibrí esmeralda hondureño (Amazilia luciae) (Figura 1) es endémico de los interiores áridos de valles de Honduras (Anderson 2014). La especie habita en el bosque espinoso árido, el cual tiene una distribución restringida dentro de Honduras. Fue considerado en peligro crítico hasta recientemente cuando fue bajado a en peligro por Birdlife International (Anderson 2014). La IUCN clasifica la especie como en peligro (EN) y su población en declive (BirdLife International 2012).

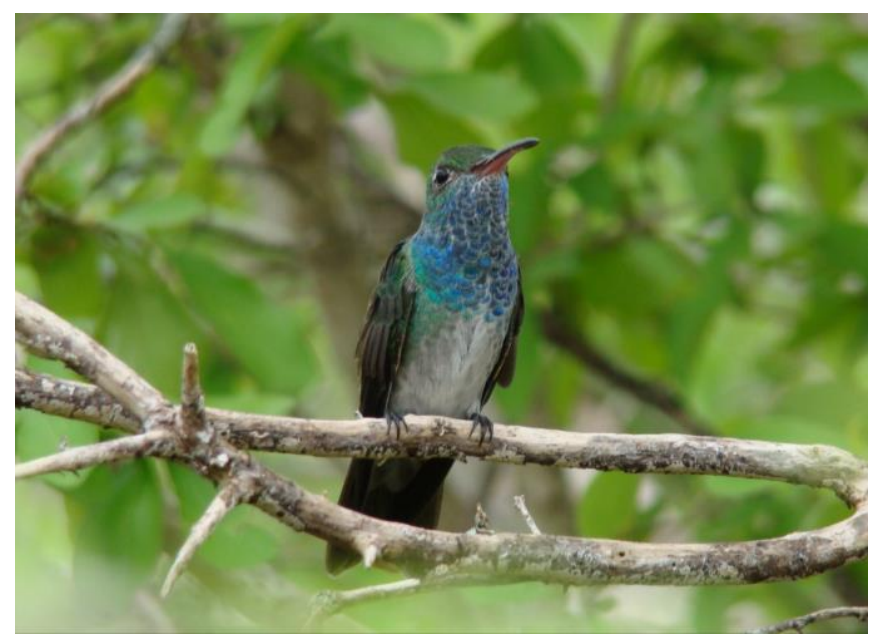

Figura 1. El colibrí esmeralda (Amazilia luciae), la única ave endémica de Honduras, existe en seis valles áridos del país incluido el valle de Agalta. Fotografía de Mario Espinal tomada el 24 de junio de 2010.

El colibrí esmeralda es poco conocido y es endémica del Bosque Seco Tropical (clasificación del sistema de Holdridge, 1967) de algunos valles áridos de Honduras. En el oriente del país se conoce de los valles de Agalta y Telica, departamento de Olancho y el valle de Aguán, departamento de Yoro. En el occidente se ha observado en los valles de Tencoa,
Quimistán y Jicatuyo en los departamentos de Santa Bárbara y Cortés (Anderson 2014). Los bosques secos del oriente son espinosos de baja altura $(5 \mathrm{~m}$ de alto en promedio) y con un dosel abierto dominado por cactus arborescentes y árboles deciduos o semideciduos. En el occidente, los bosques son más húmedos, altos y con un dosel más cerrado (Anderson et al. 2010). El Bosque Seco del valle de Agalta permanece solo como parches aislados (Mora et al. 2015).

El potencial agrícola de las tierras planas del valle de Agalta ha sido la causa de que la mayor parte del bosque seco haya sido talado y convertido en áreas agrícolas y ganaderas. Al 2012 permanecían solo 20 fragmentos de vegetación natural en buen estado de conservación. Estos se encuentran inmersos en un paisaje compuesto por una matriz de campos agrícolas y potreros de tenencia privada (Mora et al. 2015). Esta situación de tenencia de la tierra provoca la eliminación de algunos parches, el cambio de tamaño de otros o la aparición de nuevos parches de bosque en regeneración (Rodríguez et al. 2015).

El colibrí esmeralda fue descrito en 1867 y, aunque es la única ave endémica de Honduras, hasta en la década de 1980 hubo investigación aplicada para conocer más sobre ella. A partir de ahí comenzó un esfuerzo de conocimiento y protección. Por ejemplo, se paralizó un proyecto carretero en la parte superior del valle del Aguán, en el departamento de Yoro (Espinal y Mora 2012). Debido a la presencia de la especie en dicha zona, el Banco Mundial retuvo la inversión del Proyecto debido a que existía un desconocimiento casi absoluto de la distribución del ave, así como de su historia natural y los posibles impactos irremediables que la carretera podría causar al colibrí (ASESORA 2009). La paralización de esta carretera llamó la atención de la opinión pública y colocó a esta pequeña ave en una plataforma destacada. Tanto así, que un banco internacional importante la tomó en cuenta e impidió que la obra civil impactara su existencia (Espinal y Mora 2012).

La mayor parte de la investigación relacionada con 
Mora et al.: Abundancia Relativa del Colibrí Esmeralda (Amazilia luciae) en su Comunidad de Aves

A. luciae había sido desarrollada en el valle de Aguán (Espinal y Mora 2012) por lo que lo que se conocía acerca de su ecología estaba relacionado con el Bosque Muy Seco Tropical (clasificación del sistema de Holdridge, 1967). Esto hizo, que se retrasara la detección del ave en otros sitios de Bosque Seco, ya que se aducía que esta ave solo podría existir en sitios con estrictos requerimientos ecológicos como los de la parte superior del valle del Aguán. Hoy se sabe que el ave puede estar presente en bosques secos con diversos ámbitos de humedad y que su alimentación consiste de néctar de flores y artrópodos pequeños y pulpa de algunos frutos (Germer 2012).

El hábitat del colibrí esmeralda en los alrededores de Coyoles Central y Olanchito, Yoro, fue descrito en 1995 (Howell y Webb 1995). En el 2008, Espinal y Marineros (Anderson et al. 2010) descubrieron una población cerca de San José de Colinas, Santa Bárbara. Este fue el primer avistamiento de la especie en dicho departamento después de su registro en 1935 (Monroe 1968). Posteriormente, Germer (2012) publicó algunos aspectos de la biología de la especie en el bosque seco de Santa Bárbara y algunas observaciones de su comportamiento.

Debido al plan de pavimentación del tramo carretero Gualaco-San Esteban, en el Informe de Gestión Ambiental y Social (IGAS) del Banco Interamericano de Desarrollo (BID) en 2009, se estableció la necesidad de preparar una estrategia y un plan de acción para la conservación del hábitat del colibrí esmeralda en el valle de Agalta. Por esta razón se determinó la situación del colibrí esmeralda en el área de influencia del proyecto. Además, estudiamos la comunidad de aves de la zona de estudio como un posible indicador de las condiciones ecológicas generales como determinante de la supervivencia del colibrí esmeralda en el valle de Agalta.

\section{Materiales y Métodos}

Área de estudio. La descripción siguiente fue modificada de Mora et al. (2015). El valle de Agalta tiene 55,308 ha y está ubicado entre los municipios de Gualaco y San Esteban, departamento de Olancho, Honduras. El terreno es aproximadamente $45 \%$ plano y $48 \%$ ligeramente ondulado con $\pm 7 \%$ muy ondulado. El valle se encuentra bordeado al norte por la montaña de Botaderos, al sur está la sierra de Agalta, al oeste la montaña de Jacaleapa y al este la sierra del río Tinto, que sirven de barrera natural para la humedad proveniente del Caribe, lo que provoca la extrema condición seca del valle. Geográficamente, los límites del área de estudio están comprendidos entre las coordenadas 1660000 y 1692000 , al sur y norte, y 594000 y 642000 al oeste y este, respectivamente (datum WGS84; Figura 2).

En el valle de Agalta existen varias series de suelos: los de valles Tomalá, Sulaco y Danlí. El clima en el valle pertenece a las siguientes provincias climáticas. Poco Lluvioso, con Invierno Seco (Yx) que se localiza en la comunidad de Gualaco, sus meses más lluviosos son octubre y noviembre mientras que los meses más secos son febrero y marzo. Lluvioso de Altura (Vx), comprende el valle de Gualaco y la Sierra de Agalta, sus meses más lluviosos son junio y septiembre con febrero y marzo como los más secos. Muy Lluvioso de Barlovento (Ek), se localiza en San Esteban, sus meses más lluviosos son julio y septiembre y los más secos marzo y abril. En promedio se registran $1,081 \mathrm{~mm}$ de lluvia al año y la humedad relativa promedio es de $82 \%$ (Zúniga 1999).

Trabajo de campo. En agosto y septiembre de 2012 trabajamos en el valle de Agalta para determinar cuáles parches boscosos eran utilizados por el colibrí esmeralda. Además, censamos las aves en general a través de transectos de punto y observaciones libres, con énfasis en los individuos de colibrí esmeralda.

Seleccionamos al azar áreas dentro de los fragmentos de bosque para establecer los transectos de punto. Estos son una metodología que provee una manera uniforme de contar a las aves a través del tiempo y a través de sitios. Son un sistema estándar para estimar la diversidad y la abundancia de aves al contar aquellas especies vistas y observadas (Knapp y Keeley 2001). En áreas extensas, como en el valle de Agalta, los transectos de punto establecidos al azar se comportan como muestras representativas de la diversidad de aves de esa área.

En el bosque seco y ecotono realizamos 50 transectos de punto, estos entre las $0600 \mathrm{~h}$ y las $1100 \mathrm{~h}$ y de las $1400 \mathrm{~h}$ a las $1700 \mathrm{~h}$. La mayor parte de las detecciones de aves en los puntos de conteo fue de forma auditiva. Establecimos una distancia mínima de $100 \mathrm{~m}$ entre cada punto de conteo o a una distancia suficiente para no poder escuchar aves cantando en un punto anterior. En cada sitio, el transecto tuvo una 
duración de 10 minutos durante los cuales anotamos cada especie vista o escuchada. Esperamos 2 minutos antes de comenzar cada transecto para dar tiempo a que las aves se habituaran a nuestra presencia. Detectamos unas especies fuera del esquema de transectos por lo que no las incluimos en el análisis de abundancia. No tomamos en cuenta a la paloma de Castilla o doméstica (Columba livia) ni al gorrión europeo (Passer domesticus) por ser aves introducidas. Estas especies son muy abundantes en el área urbana de San Esteban.

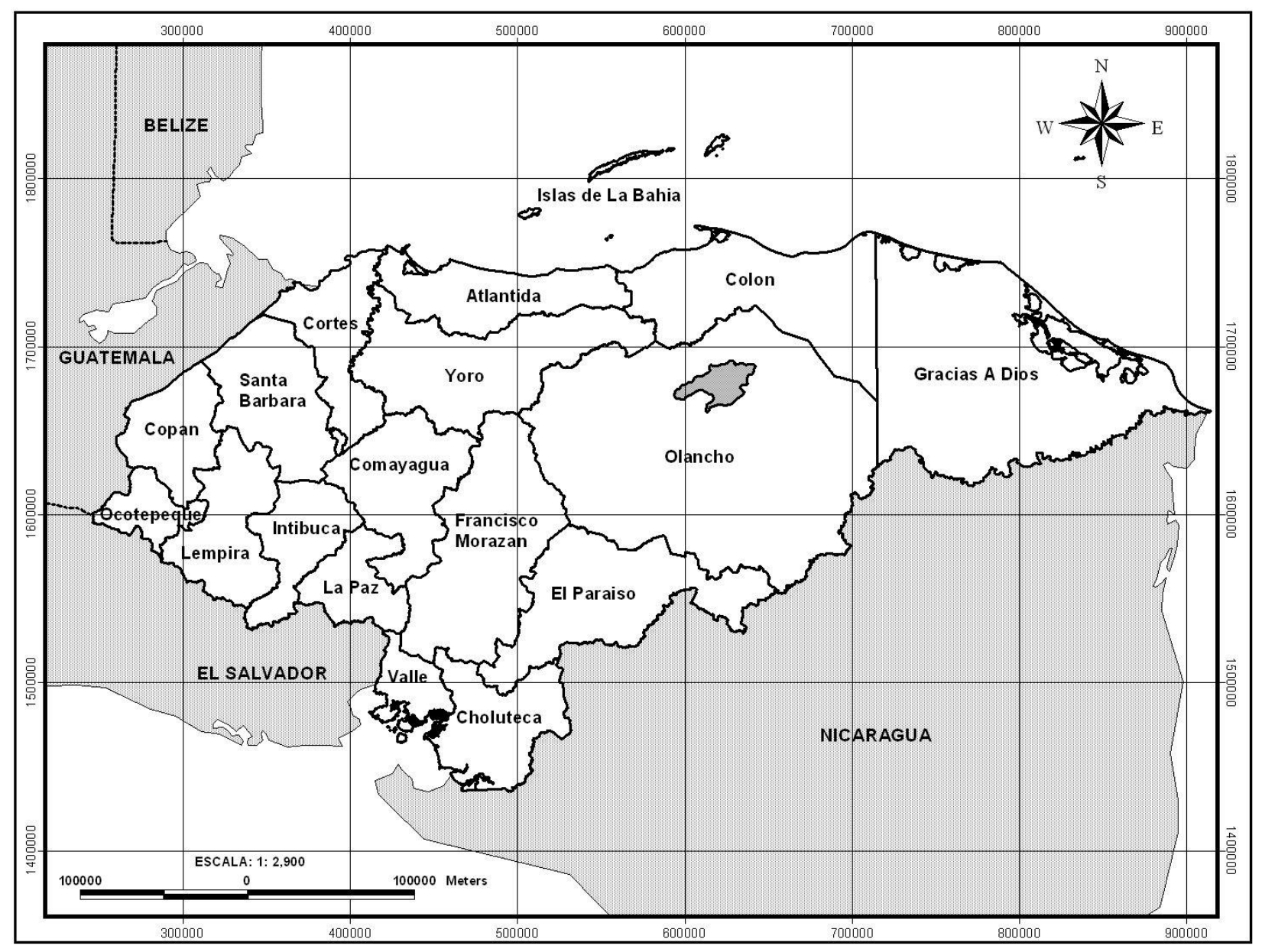

Figura 2. Valle de Agalta, en gris dentro del departamento de Olancho, Honduras.

Como cualquier otro método de muestreo, los transectos de punto para abundancia tienen sesgos. El sesgo es causado por la dificultad de detectar todas las especies presentes en el área muestreada. Para reducir el sesgo, redujimos el área efectiva máxima de detección a $25 \mathrm{~m}$ (Hutto et al. 1986). Esto permite detectar con mayor eficiencia las aves presentes dentro del área aunque se sacrifica la posibilidad de detectar mayor diversidad. No obstante, en nuestro caso, el tamaño de la muestra compensa la reducción de la diversidad ya que aumentamos el número de sitios. Con esto aumentamos la posibilidad de detectar especies crípticas. Asimismo, al aumentar el tamaño de la muestra, redujimos el sesgo de la baja probabilidad de detección de especies comunes crípticas o de especies raras de difícil detección. La 
Mora et al:: Abundancia Relativa del Colibrí Esmeralda (Amazilia luciae) en su Comunidad de Aves

frecuencia de detección estimada de los transectos de punto sin determinación de distancia o muestreo simple son muy útiles en estudios comparativos a largo plazo (Blondel et al. 1981).

Los ámbitos de abundancia los obtuvimos según los criterios de Bibby et al. (1998). Una especie abundante es aquella registrada dentro del hábitat apropiado en más de $35 \%$ de los transectos; común la registrada entre 12 y $33 \%$ de los transectos; frecuente es la registrada en al menos tres transectos y las especies raras son aquellas registradas menos de tres veces en los transectos o detectadas fuera del hábitat normal de la especie. Los transectos de punto proveen una tasa de esfuerzo/encuentro. Así que cada especie detectada en 17 o más transectos la consideramos abundante, entre 6 y 16 común, entre tres y cinco frecuente y en dos o menos rara (Bibby et al. 1998).

A través de la modificación de los métodos de conteo se puede estimar la densidad de las especies de aves (Reynolds et al. 1980). No obstante, la aplicación de estos es compleja y sujeta a muchos sesgos (Verner y Ritter 1985). Debido a ello no intentamos determinar la densidad de las especies, ya que una premisa para esto es la repetición y comparación de los puntos a través de un tiempo determinado (Beals 1960).

Cuadro 1. Especies de aves detectadas, con su respectivo estado y abundancia, en el valle de Agalta Olancho, Honduras, 2012.

\begin{tabular}{llll}
\hline Nombre científico & Nombre común & Estado & Abundancia \\
\hline Dendrocygna autumnalis & Piche & Residente & DF$^{\S}$ \\
\hline Ortalis vetula & Chachalaca & Residente & Rara \\
\hline Colinus cristatus & Codorniz, chochorica & Residente & Común \\
\hline Tachybaptus dominicus & Zambullidor & Residente & DF \\
\hline Mycteria americana & Cigüeña & Residente & DF \\
\hline Ardea herodias & Garzón azul & Migratorio & DF \\
\hline Ardea alba & Garzón blanco & Residente & DF \\
\hline Bubulcus ibis & Garcita bueyera & Residente & DF \\
\hline Platalea ajaja & Garza rosada & Residente & DF \\
\hline Coragyps atratus & Zope & Residente & DF \\
\hline Cathartes aura & Tincute & Residente & DF \\
\hline Chondrohierax uncinatus & Milano pico ganchudo & Residente & Raro \\
\hline Elanus leucurus & Milano cola blanca & Residente & DF \\
\hline lctinia plumbea & Milano plúmbeo & Residente & Raro \\
\hline Buteo magnirostris & Gavilán de camino & Residente & Común \\
\hline Buteo plagiatus & Gavilán gris & Residente & DF \\
\hline Buteo brachyurus & Gavilán de cola corta & Residente & DF \\
\hline Buteo albonotatus & Gavilán tincute & Residente & Raro \\
\hline Caracara cheriway & Caracara & Residente & DF \\
\hline Herpetotheres cachinnans & Guaco & Residente & Frecuente \\
\hline Falco sparverius & Halcón lis-lis & Residente & DF \\
\hline Actitis macularius & Alzacolita & Migratorio & Raro \\
\hline Jacana spinosa & Gallito de agua & Residente & DF \\
\hline
\end{tabular}


Cuadro 1. Continuación

\begin{tabular}{|c|c|c|c|}
\hline Nombre científico & Nombre común & Estado & Abundancia \\
\hline Patagioenas flavirostris & Azulona & Residente & DF \\
\hline Zenaida asiática & Paloma ala blanca & Residente & Frecuente \\
\hline Columbina inca & Turquita coluda & Residente & Común \\
\hline Columbina passerina & Turquita empedrada & Residente & Raro \\
\hline Columbina talpacoti & Turquita rojiza & Residente & Raro \\
\hline Leptotila verreauxi & Paloma barranquera & Residente & Raro \\
\hline Aratinga holochlora & Perico ocotero & Residente & Raro \\
\hline Aratinga nana & Perico comejenero & Residente & Raro \\
\hline Amazona albifrons & Lora de frente blanca & Residente & Raro \\
\hline Piaya cayana & Pájaro león, Luis & Residente & Frecuente \\
\hline Crotophaga sulcirostris & Tijul & Residente & Abundante \\
\hline Geococcyx velox & Correcaminos & Residente & Raro \\
\hline Glaucidium brasilianum & Búho picapiedra & Residente & Común \\
\hline Cypseloides niger & Vencejo & Migratorio & Raro \\
\hline Chlorostilbon canivetii & Gorrión & Residente & Frecuente \\
\hline Amazilia luciae & Colibrí esmeralda & Residente & Común \\
\hline Amazilia rutila & Colibrí canelo & Residente & Abundante \\
\hline Momotus coeruliceps & Taragón & Residente & Raro \\
\hline Eumomota superciliosa & Taragón & Residente & Común \\
\hline Melanerpes formicivorus & Checo ocotero & Residente & Frecuente \\
\hline Melanerpes aurifrons & Chengue & Residente & Común \\
\hline Colaptes rubiginosus & Checo verdoso & Residente & Raro \\
\hline Dryocopus lineatus & Checo cabeza roja & Residente & Raro \\
\hline Thamnophilus doliatus & Hormiguero & Residente & Común \\
\hline Lepidocolaptes souleyetii & Trepatronco & Residente & Raro \\
\hline Elaenia flavogaster & Mosquero & Residente & Raro \\
\hline Oncostoma cineiregulare & Mosquero pico torcido & Residente & Frecuente \\
\hline Empidonax traillii & Mosquerito & Migratorio & Frecuente \\
\hline Empidonax minimus & Mosquerito & Migratorio & Raro \\
\hline Myiarchus tuberculifer & Mosquero llorón & Residente & Frecuente \\
\hline Pitangus sulphuratus & Cristofué, bicho feo & Residente & Común \\
\hline Megarynchus pitangua & Cristofué, bicho feo & Residente & DF \\
\hline Myiozetetes similis & Cristofué pequeño & Residente & DF \\
\hline Myiodinastes luteiventris & Mosquero & Migratorio & Raro \\
\hline Tyrannus melancholicus & Chilero & Residente & Frecuente \\
\hline Tyrannus savana & Tijerilla & Residente & Raro \\
\hline
\end{tabular}


Mora et al:: Abundancia Relativa del Colibrí Esmeralda (Amazilia luciae) en su Comunidad de Aves

Cuadro 1. Continuación

\begin{tabular}{|c|c|c|c|}
\hline Nombre científico & Nombre común & Estado & Abundancia \\
\hline Tityra semifasciata & Torreja & Residente & Raro \\
\hline Vireo griseus & No hay nombre común & Migratorio & Raro \\
\hline Cyanocorax melanocyaneus & Cerequeque & Residente & Frecuente \\
\hline Progne chalybea & Golondrina & Residente & Común \\
\hline Hirundo rustica & Golondrina & Migratorio & Común \\
\hline Thryothorus maculipectus & Cucarachero & Residente & Común \\
\hline Cantorchilus modestus & Cucarachero & Residente & Frecuente \\
\hline Troglodyes aedon & Cucarachero & Residente & DF \\
\hline Polioptila albiloris & Monjita & Residente & Común \\
\hline Turdus grayi & Zorzal & Residente & Frecuente \\
\hline Mimus gilvus & Sinzontle & Residente & Raro \\
\hline Oreothlypis peregrina & Chipe & Migratorio & Raro \\
\hline Geothlypis poliocephala & Chipe & Residente & Frecuente \\
\hline Setophaga ruticilla & Chipe & Migratorio & Raro \\
\hline Setophaga magnolia & Chipe & Migratorio & DF \\
\hline Setophaga petechia & Chipe amarillo & Migratorio & Frecuente \\
\hline Basileuterus rufifrons & Chipe & Residente & Raro \\
\hline Thraupis abbas & Viuda & Residente & DF \\
\hline Saltator coerulescens & Chorcha gris & Residente & DF \\
\hline Saltator atriceps & Chorcha loca & Residente & Frecuente \\
\hline Volatinia jacarina & Semillerito & Residente & Frecuente \\
\hline Sporophila torqueola & Semillerito & Residente & Raro \\
\hline Tiaris olivaceus & Semillerito & Residente & Raro \\
\hline Arremonops chloronotus & Pinzón lomo verde & Residente & Común \\
\hline Cyanocompsa parellina & Piquigrueso azul & Residente & Raro \\
\hline Sturnella magna & Chira, pradero & Residente & DF \\
\hline Dives dives & Guachir & Residente & Raro \\
\hline Quiscalus mexicanus & Zanate, clarinero & Residente & Frecuente \\
\hline Icterus pectoralis & Chorcha & Residente & Frecuente \\
\hline Icterus gularis & Chorcha & Residente & Raro \\
\hline Euphonia affinis & No hay nombre común & Residente & Raro \\
\hline
\end{tabular}

${ }^{\S} \mathrm{DF}=$ detecciones únicas o por fuera del sistema de transectos de punto. 


\section{Resultados}

Junto con el colibrí esmeralda (Figura 1), detectamos 89 especies de aves de 36 familias en el valle de Agalta (Cuadro 1). Estas especies las detectamos en los fragmentos de bosque seco identificados con anterioridad (Mora et al. 2015), el ecotono de pino-roble, los sistemas agropecuarios y los bosques de galería dentro del bosque seco. Al $58 \%$ de las especies observadas en Agalta (64 de 90) las detectamos en los transectos de punto. De estas, 3\% son abundantes, $22 \%$ son comunes y $25 \%$ son frecuentes (Cuadro 1). El otro 50\% fueron especies raras, un porcentaje alto debido a que varias de las especies detectadas son migratorias y apenas comenzaban a llegar. Otras especies las detectamos solo en el ecotono de pino-roble, un hábitat donde invertimos un menor esfuerzo de muestreo.

Detectamos al colibrí esmeralda en nueve fragmentos de los 20 identificados en el valle de Agalta (Figura 3). Estos fueron los fragmentos 4, 8, 9, 10, 11, $14,15,16$ y 17 (Figura 3). La especie fue más común en el fragmento de bosque del botadero municipal de San Esteban (Fragmento 15, Figura 3). Aquí hicimos el mayor número de detecciones y observaciones de eventos de defensa de territorios por el colibrí esmeralda.

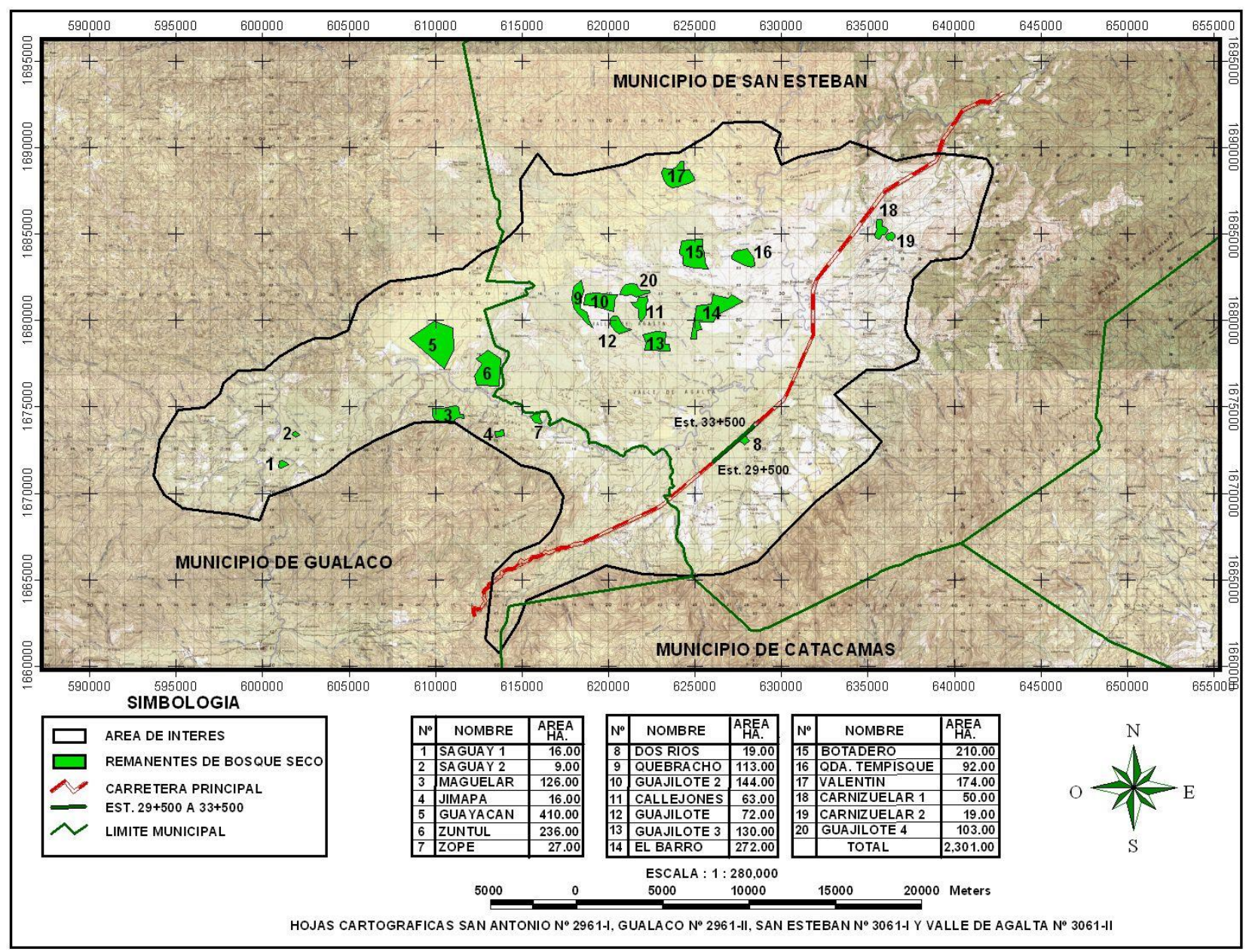

Figura 3. Remanentes o parches de Bosque Seco Tropical identificados y delimitados en el valle de Agalta, Olancho, Honduras, por Mora et al. (2015). 
Observamos a Amazilia luciae libar néctar de al menos cuatro especies de plantas, Combretum fruticosum, Pilosocereus chrysacanthus, Lantana camara y Psittacanthus sp. (Figura 4). En los predios y dentro del bosque del botadero municipal observamos colibríes recogiendo insectos en telas de araña. Algunas de las detecciones del colibrí fueron en sitios abiertos (Figura 5). Lo detectamos a $617 \mathrm{msnm}$ lo que representa un punto de elevación máxima en el oriente de la distribución de la especie (se ha detectado a 660 msnm en el departamento de Santa Bárbara, Germer 2012). El punto de menor altitud donde observamos al colibrí esmeralda en el valle de Agalta fue a 475 msnm.
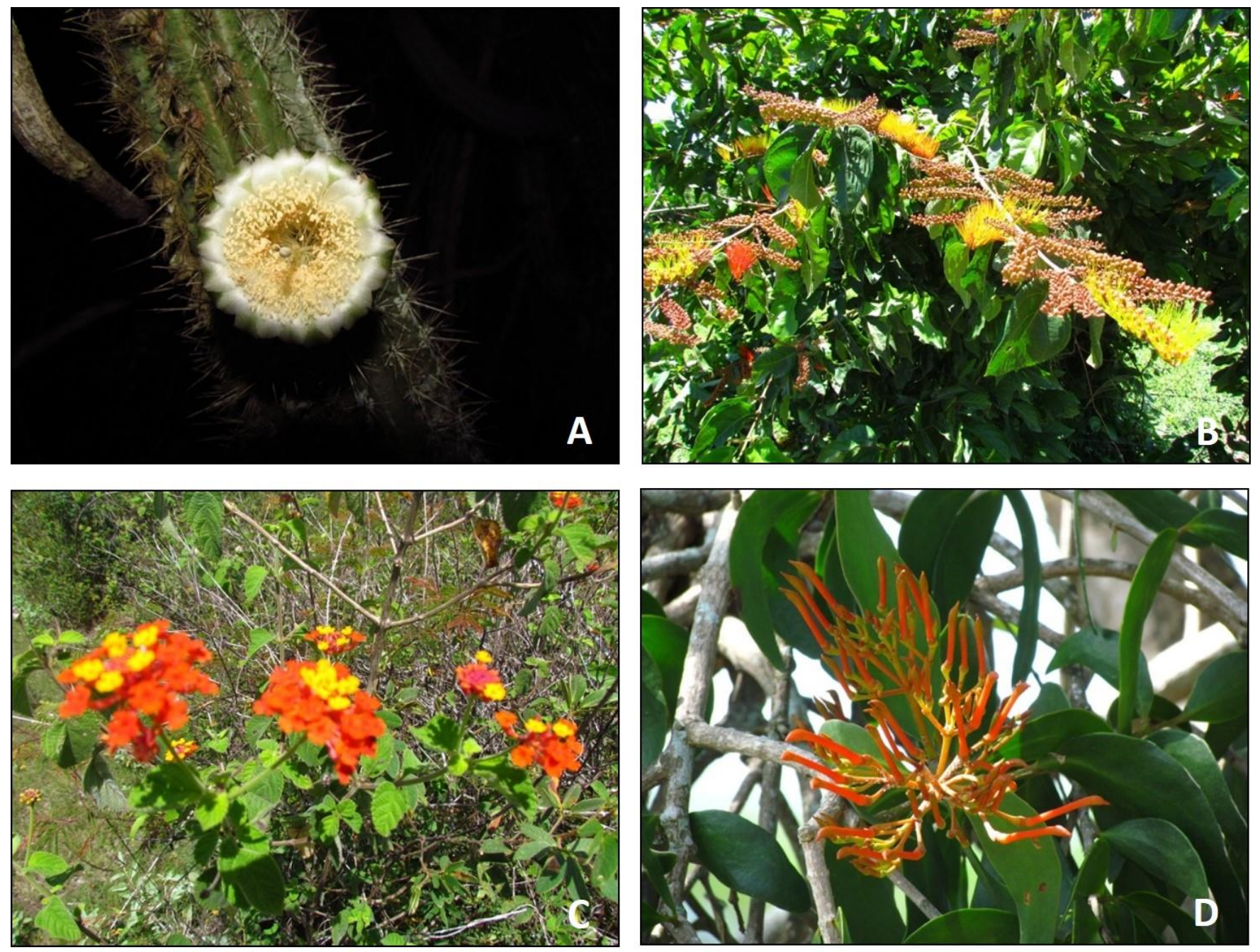

Figura 4. Especies de plantas donde se observó al colibrí esmeralda (Amazilia luciae) libar néctar en el valle de Agalta. A. Pilosocereus chysacanthus (Cactaceae), B. Combretum fruticosum (Combretaceae), C. Lantana camara (Verbenaceae), D. Psittacanthus sp. (Loranthaceae).

\section{Discusión}

La gran mayoría de las aves observadas son generalistas. La única especie especialista observada fue el colibrí esmeralda ya que solo existe en bosques secos (Germer 2012, Germer et al. 2013). Solamente el colibrí esmeralda está dentro de alguna categoría de amenaza pues está clasificado como en peligro (Bird 
International 2012). Esto se debe a que la especie se encuentra en seis localidades restringidas a los interiores secos de los valles de Agalta y Telica en Olancho, el valle de Aguán en Yoro, Tencoa y Quimistán en Santa Bárbara y Jicatuyo en Cortés (Anderson 2014). El resto de las especies observadas tienen poblaciones estables. No obstante, Arremonops chloronotus twomeyi solo existe en los valle áridos de Agalta y Aguán. Esta población está aislada y los individuos son más pequeños que sus congéneres del resto del país (Monroe 1968, Howell y Webb 1995, Bonta y Anderson 2002).

Cuarenta y un especies fueron detectadas en los mismos transectos junto con Amazilia luciae. Amazilia rutila, Crotophaga sulcirostris, Eumomota superciliosa, Melanerpes aurifrons, Buteo magnirostris y Glaucidium brasilianum fueron las más frecuentes (Cuadro 1). Las especies categorizadas como abundantes fueron Crotophaga sulcirostris y Amazilia rutila, que es probablemente el colibrí más común del país. Por el contrario, Chondrohierax uncinatus y Cypseloides niger fueron las más raras (Cuadro 1) y son generalmente difíciles de detectar (Bonta y Anderson 2002). Sin duda el número de especies en el valle de Agalta es mucho mayor. Esas especies se detectarían en un estudio a más largo plazo. De hecho en un estudio de un año realizado recientemente se detectaron 176 especies de aves el valle de Agalta (Rodríguez et al. 2015).

El ecotono de pino-roble en el área de estudio está a corta distancia de un banco de préstamo para el mantenimiento de la carretera Gualaco-San Esteban (Figura 5). Maquinaria pesada cruza por el sitio donde observamos al colibrí esmeralda por lo que aparentemente, los altos niveles de ruido no lo perturba ya que incluso un macho defendía su territorio a la orilla de la carretera (Figura 5). La protección de este territorio por dicho macho implica que el ave utiliza de manera activa esta área probablemente rotando entre el ecotono y el bosque seco inmediato.

El resto de las detecciones de Amazilia luciae las obtuvimos en el bosque seco. No obstante, no observamos individuos dentro del bosque. En todas las detecciones en bosque seco, las aves se movían contiguas a áreas abiertas. La mayor cantidad de colibríes los observamos en los remanentes asociados al botadero municipal de San Esteban (Parche 15, figura 3). Esto concuerda con modelaciones de abundancia hechas recientemente según los datos recopilados en un estudio de un año en el sitio (Rodríguez el al. 2015). El resto de las detecciones de Amazilia luciae en Agalta fueron periféricas al remanente del botadero municipal de San Esteban. En Agalta este colibrí parece preferir las orillas del bosque que generalmente se encuentran adyacentes a caminos, potreros, pastizales y áreas de cultivo.
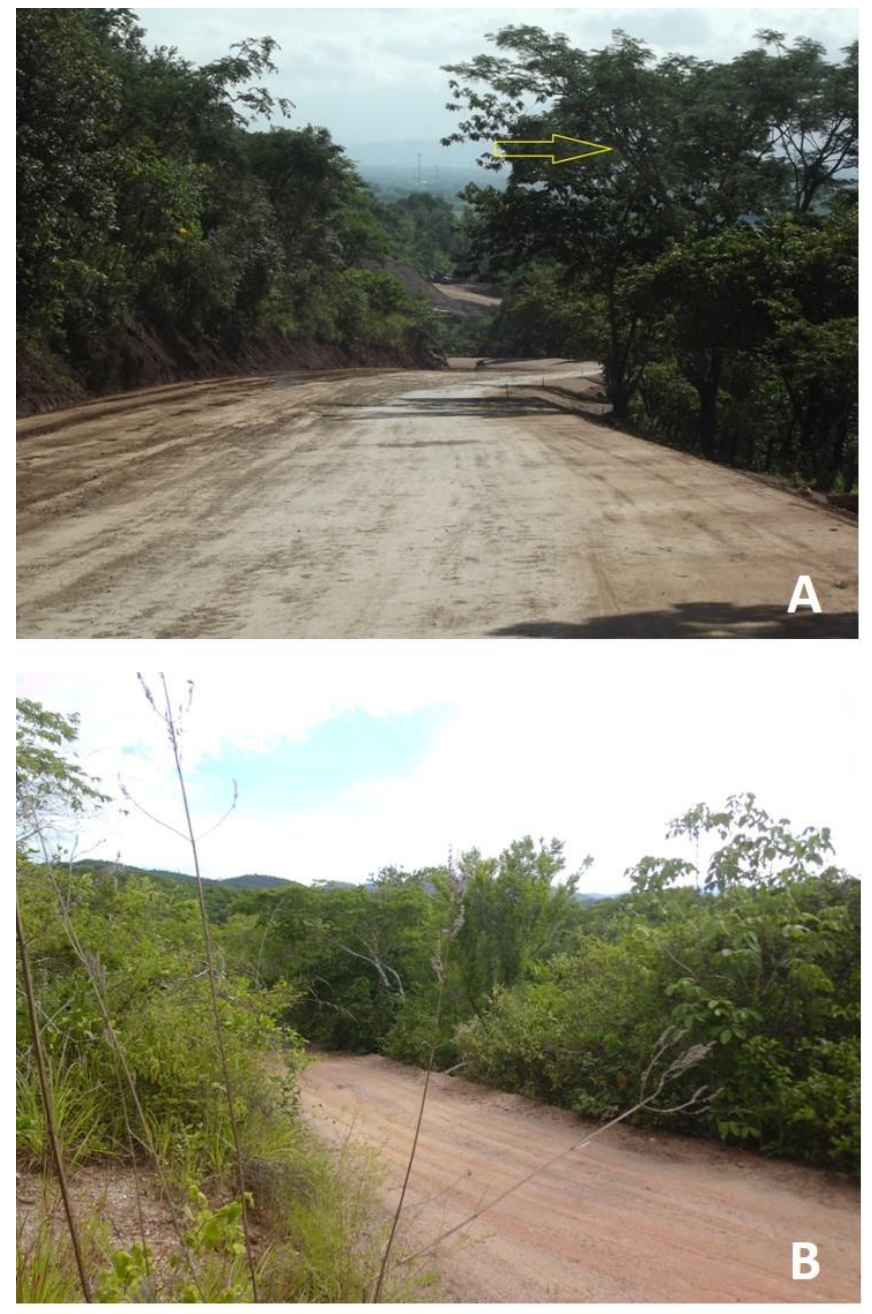

Figura 5. A. Banco de préstamo de material en el que se detectó la presencia de Amazilia luciae. La flecha amarilla señala el árbol en que se observó a un macho defender su territorio. B. Transecto de Punto No. 12 en el cual se detectó Amazilia luciae, nótese que es un área abierta. 
Mora et al.: Abundancia Relativa del Colibrí Esmeralda (Amazilia luciae) en su Comunidad de Aves

Categorizamos a Amazilia luciae (Figura 1) como común dado que lo observamos al menos una vez en cada día de muestreo. A pesar de esto, no se podría decir que es abundante ya que, comparada con una especie bajo esta categoría, tenemos menos observaciones de colibrí. Detectamos al colibrí en 16 ocasiones durante los muestreos, 19 individuos en el área de muestreo. No obstante, para determinar la cantidad real de colibríes en el valle de Agalta, se necesita una investigación a largo plazo con ese propósito. El resultado de común resulta en un estado de conservación suficientemente positivo para poder comenzar un programa de conservación. Aunque en Agalta Amazilia luciae resultó relativamente común, cumple con al menos dos de los criterios de rareza (distribución mundial restringida, hábitat restringido y baja densidad poblacional, Rabinowitz 1981).

Para la conservación del colibrí esmeralda en Agalta hay que proteger el hábitat. La categoría VI de IUCN de manejo de especie/hábitat puede ser una opción. No obstante, la creación de un área protegida con este objetivo es muy complicada ya que la mayoría de fragmentos de bosque seco en Agalta están en manos privadas (Mora et al. 2015). Una alternativa válida podría ser la creación de un área protegida privada, la cual dependería de los beneficios económicos que su propietario tendría por su creación.

Dos áreas ofrecen buenas posibilidades para la creación de un área protegida privada debido a sus tamaños y a sus posibilidades de establecimiento. El parche Guayacán (Figura 3) es de un relativo buen tamaño (410 ha) y el terreno donde está el botadero municipal es de propiedad del municipio. Esta propiedad podría ser transformada y manejada como área de conservación del colibrí esmeralda. A la vez, el municipio podría destinar esta reserva como parte de un proyecto de turismo rural o científico en la zona en la que además existen otros atractivos (Espinal y Mora 2012). Permitir que los potreros en desuso se recuperen es una solución temporal si la tierra sigue en manos privadas. Más crítico aún es que la dinámica de los parches de vegetación es cambiante en el valle de Agalta. Sitios o parches con vegetación en 2012 (Mora et al. 2015) fueron modificados a potreros mediante el uso de tractores que removieron árboles, cactus y arbustos (Rodríguez el al. 2015).

Hay que tomar en cuenta que para la conservación del colibrí esmeralda en el valle de Agalta, los fragmentos no son unidades individuales de conservación, ya que el colibrí aparentemente se desplaza según la concentración de alimento. Por lo tanto, se debe incrementar la conectividad entre los fragmentos mediante el aprovechamiento de los bosques de galería. Estos, así como algunas de las propiedades con fragmentos de bosque seco podrían ser conservados bajo un programa de pago por servicios ambientales. Posiblemente, esta es la alternativa más válida para la conservación del colibrí esmeralda en la valle de Agalta ya que puede conservar el hábitat remanente aunque esté en manos privadas. Quien sea el ente encargado del establecimiento de un programa tal en la zona debe buscar alternativas posibles para su éxito. El aspecto clave sin duda es el financiero aparte de la educación ambiental y otras actividades acompañantes. Un peaje ambiental en la carretera en el tramo Gualaco-San Esteban podría generar al menos fondos parciales para dicho programa (Espinal y Mora 2012).

\section{Literatura Citada}

Anderson, D.L. 2014. Honduran Emerald (Amazilia luciae), Neotropical Birds Online. T. S. Schulenberg, Editor. Ithaca, Cornell Lab of Ornithology. Consultado 16 de julio de 2016. Disponible en: http://neotropical.birds.cornell.edu/portal/species/overvie w?p $p$ spp $=252731$

Anderson, D.L., P. House, R.E. Hyman, R. Steiner, H.R. Hawkins, S. Thorn, M.J. Rey, M.R. Espinal y L.E. Marineros. 2010. Rediscovery of the Honduran Emerald Amazilia luciae in western Honduras: insights on the distribution, ecology, and conservation of a Critically Endangered' hummingbird. Bird Conservation International. BirdLife International. 8 p.

ASESORA, S. de R.L. 2009. Plan de manejo del área hábitat/especie del colibrí esmeralda hondureño. 153 p.

Beals, E. 1960. Forest bird communities in the Apostle Islands of Wisconsin. Wilson Bulletin 72:156-181.

Bibby, C., M. Jones y S. Marsden. 1998. Expedition Field Techniques: Bird Surveys. Expedition Advisory Centre, Royal Geographic Society. London. 143 p.

BirdLife International. 2012. Amazilia luciae. The IUCN Red List of Threatened Species 2012: e.T22687529A40515217. Disponible en: http://dx.doi.org/10.2305/IUCN.UK.20121.RLTS.T22687529A40515217.

Blondel, J., C. Ferry y B. Frochot. 1981. Point counts with unlimited distance. In: C.J. Ralph y J.M. Scott (eds.). Estimating numbers of terrestrial birds. Studies in Avian Biology 6: 414-420. 
Bonta, M. y D. Anderson. 2002. Birding Honduras A Checklist and Guide. Ecoarte S. de R.L. Tegucigalpa M.D.C. 186 p.

Espinal, M.R. y J.M. Mora. 2012. Estrategia de conservación del colibrí esmeralda (Amazilia luciae) en el valle de Agalta. BID/SOPTRAVI/Dirección General de Carreteras/Cuenta del Desafío del Milenio, Honduras/ICF. Honduras.

Germer, L.D. 2012. Observaciones Puntuales de Colibrí Esmeralda Amazilia luciae (Trochilidae) en el Bosque Seco Intermontano del Departamento de Santa Bárbara durante los meses de Abril y Mayo del 2011. El Esmeralda 1(1):52-64.

Germer, L.D., A. Alvarado, E., Miranda, I. Miranda y W. Orellana. 2013. Descubrimiento de Amazilia luciae (Trochilidae) en el departamento de Lempira. El Esmeralda 2(2):91-93.

Holdridge, L.R. 1967. Life zone ecology. Tropical Science Center. San José, Costa Rica. 206 p.

Howell, S. y S. Webb. 1995. A guide to the Birds of Mexico and Northern Central America. Point Reyes Bird Observatory, Oxford University Press. 1,010 p.

Hutto, R., S. Pletschet y P. Hendricks. 1986. A Fixed Radius Point Count Method for Non-Breeding and Breeding Use. The Auk 103:593-602.

INGTELSIG. 2011. Descripción de los Remanentes de Hábitat del Colibrí esmeralda, Departamento de Santa Bárbara, Honduras. SERNA-ICF-EPR. 104 p.

Knapp, E. y J. Keeley. 2001. A National Study of the Consequences of Fire and Fire Surrogate Treatments. Sequoia National Park Site Study Plan. 37 p.
Monroe, B.L., Jr. 1968. A distributional Survey of the Birds of Honduras. Ornithological Monographs No. 7 American Ornithologists' Union, Anchorage, KY. 458 p.

Mora, J.M., M.R. Espinal, L.I. López y B.O. Quezada. 2015 [2012]. Caracterización del Bosque Seco Tropical Remanente en el Valle de Agalta, Honduras. Ceiba 53(1):38-56.

Rabinowitz, D. 1981. Seven forms of rarity. In: H. Synge (Ed.). The Biological Aspects of Rare Plant Conservation. John Wiley \& Sons Ltd. Chichester, New York, Brisbane, Toronto. p. 205-217.

Rodríguez, F., J. Larkin, S. Cruz, T. Mejía, L. Ferrufino y J. Townsend. 2015. Evaluación de Biodiversidad y Ecosistema del Valle de Agalta para la Protección del Colibrí Esmeralda (Amazilia luciae). Reporte final. IUP. $\mathrm{RI} \&$ UNAH. Honduras. $93 \mathrm{p}$.

Verner, J. y L.V. Ritter. 1985. A comparison of transects and point counts in oak-pine woodlands of California. Condor 87:47-68.

Vreugdenhil, D., P.R. House, C.A. Cerrato, R.A. Martínez y A.C. Pereira. 2002. Racionalización del sistema nacional de las Áreas Protegidas de Honduras. Volumen 1: Estudio Principal. PPROBAP/Banco Mundial/UNDP/GEF /WICE. 54 p.

Zúniga, E. 1999. Mapa de Clasificación Climática de Honduras. Escala 1:1, 000,000. Tegucigalpa M.D.C., Honduras.

Recibido para publicación el 28 de abril de 2016.

Aceptado para publicación el 1 de octubre de 2016. 